\title{
A SIMPLE AND EFFECTIVE RIGID BEAM MODEL FOR STUDYING THE DYNAMIC BEHAVIOUR OF FREESTANDING COLUMNS
}

\author{
DANIELE BARALDI ${ }^{*}$, GABRIELE MILANI ${ }^{2}$ AND VASILIS SARHOSIS ${ }^{3}$ \\ ${ }^{1}$ Università IUAV di Venezia \\ Terese, Dorsoduro 2206, 30123, Venezia, Italy \\ e-mail: danielebaraldi@iuav.it, http://www.iuav.it (*corresponding author) \\ ${ }^{2}$ Department of Architecture, Built Environment and Construction Engineering \\ Technical University of Milan \\ Piazza Leonardo Da Vinci 32, 20133, Milano, Italy \\ e-mail: gabriele.milani@polimi.it, http://www.abc.polimi.it \\ ${ }^{3}$ School of Civil Engineering \\ University of Leeds \\ LS2 9JT, Leeds, UK \\ e-mail: V.Sarhosis@leeds.ac.uk, https://engineering.leeds.ac.uk
}

Keywords: Masonry, Columns, DEM, Rigid Beam Model, Dynamic response.

\begin{abstract}
In this work, a simple and effective Rigid Beam Model is proposed for studying the dynamic behaviour of ancient freestanding stone columns. As well known, monolithic and multi-drum freestanding columns are historical structural elements typical of ancient temples that still can be found in the Mediterranean area. These columns are particularly prone to collapse in case of seismic actions. The dynamic behaviour of freestanding columns has been studied by many authors in the past [1-3], and it is characterized by a strong nonlinearity due to sliding and rocking between the drums. The Rigid Beam Model here described assumes each drum of the column as a rigid beam element and each interface between the drums as a node of the model able to move horizontally. Similar to Housner [1] approach, this model assumes small displacements and no sliding between blocks. Furthermore, the material nonlinearity is considered by means of a moment-rotation constitutive law slightly modified with respect to the bilinear one introduced by Housner. Numerical simulations were performed on monolithic and multi-drum columns modelled using the proposed Rigid Beam Model approach. Results are compared against simulations of the same columns undertaken by means of the Discrete Element Model, which is able to consider large displacements and the possible sliding between the drums, and it has already proven its effectiveness in simulating column behaviour [2-4].
\end{abstract}

\section{INTRODUCTION}

Monolithic and multi-drum stone columns are structural elements typical of ancient monuments of the Mediterranean area, built, among the others, by Roman, Greek, and Egyptian civilizations. In the current days, such columns can be often found in freestanding condition, given that the original buildings had been fully or partially destroyed by past earthquakes and other natural or anthropic events. Even if these structural elements survived up to current days, 
they are still subjected to seismic actions and they are prone to collapse.

The analytical and numerical assessment of the dynamic behavior of monolithic and multi-drum columns was studied by many researchers (see for instance the references collected in [4]). In particular, the pioneering analytical model proposed by Housner [1] is the first contribution that studied the behavior and the possible overturning of a single rigid block subjected to different types of horizontal actions. The subsequent research activities focused on the assessment of the dynamic behavior of monolithic elements by means of accurate numerical models and with laboratory experiments. Some other research activities focused on the analytical assessment of the dynamic behavior of columns made of a limited number of drums, whereas most of the research activities were dedicated to the use of more or less accurate numerical models for simulating the dynamic behavior of multi-drum columns and more complex stone structures (namely buttresses, arches on columns, trilithic structures). Among the different numerical models adopted for studying monolithic and multi-drum columns, the Discrete Element Method (DEM), introduced for the analysis of soils and rocks [6], has been extensively used, due to the possibility of modelling the column drums as distinct elements, with the possibility to apply dynamic excitations and achieve large displacements.

In this work, a simple and effective numerical Rigid Beam Model, already introduced by authors [5] for studying the dynamic behavior of both monolithic and multi-drum columns, is further investigated. The model considers a multi-drum column as an assemblage of vertically aligned rigid beam elements, with each drum represented by a beam element and each interface

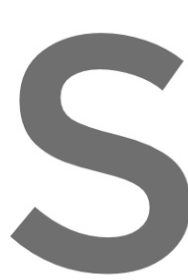
represented by a node. Small displacements interface level. The simulating the numerica Spanos and Koh [7], and one of the solutions proposed by Housner [1] from harmonic analyses on slender monolith
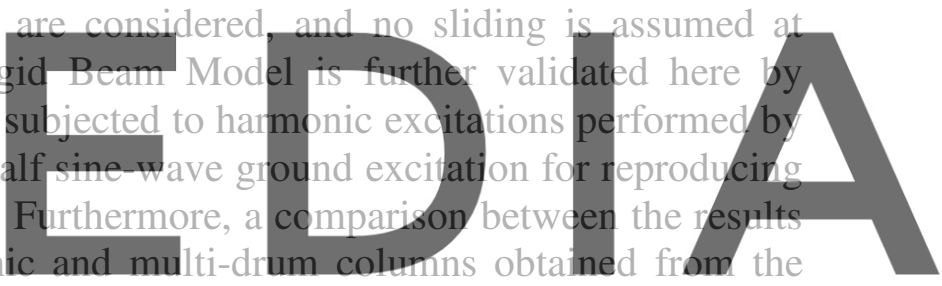

Rigid Beam Model and the two-dimensional (2D) Discrete Element (DE) code UDEC [8] are

\section{RIGID BEAM MODEL}

As stated in the introduction, the Rigid Beam Model was introduced by authors in a recent contribution [5] for representing a generic multi-drum column made of $n$ blocks or drums on a rigid ground, having overall height $H$, lower base width or diameter $B_{1}$ and upper width or diameter $B_{n}$ (Figure 1a). The rigid beam model is defined by assuming a rigid beam element for each drum of the column and considering $n+1$ nodes at beam element ends, representing the interfaces between the drums (Figure 1b). Sliding between the drums is neglected; hence, the degrees of freedom of the rigid beam model are given by horizontal nodal translations, velocities and accelerations $u_{i}, \dot{u}_{i}$ and $\ddot{u}_{i}$, respectively (Figure 1b). Small displacements are also assumed, hence a generic beam element can be subjected to a rigid rotation depending only on upper and lower nodal displacements and element height: $\theta_{i}=\left(u_{i+1}-u_{i}\right) / h_{i}$. Each beam element is also characterized by a mass $m_{i}$ and by the corresponding weight $P_{i}$. 


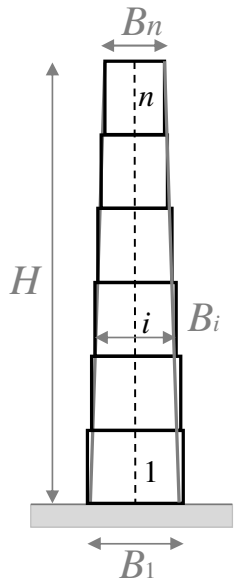

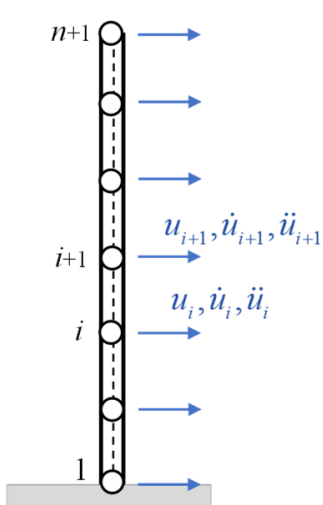

b

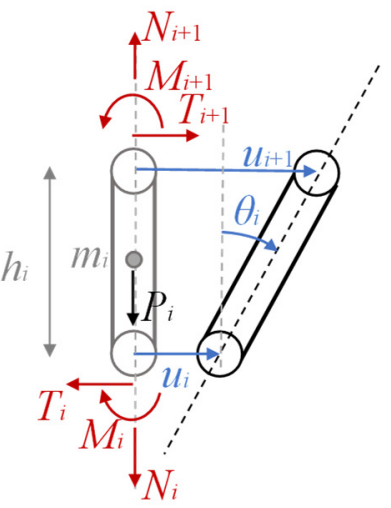

C

The typical internal forces of the beam element, namely a normal force, a shear force, and a moment $N_{i}, T_{i}$, and $M_{i}$, respectively, act at each beam end (Figure 1c). The translational and rotational equations of motion for a beam element may be written as follows:

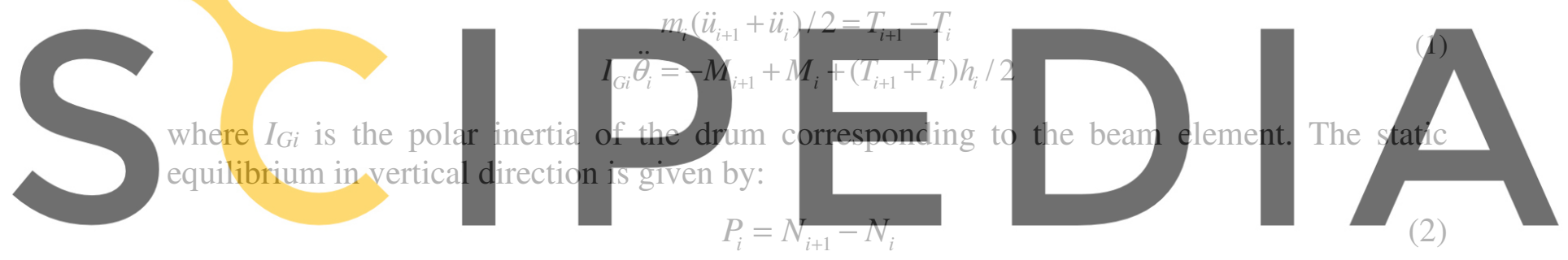

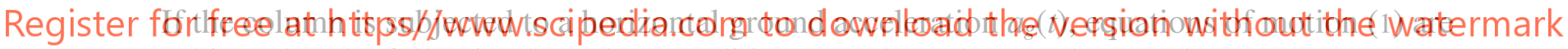
subjected to the following boundary conditions at column base and top nodes:

$$
\begin{gathered}
\ddot{u}_{1}=\ddot{u}_{g}=a_{g}(t) \\
T_{n+1}=0 \\
M_{n+1}=0
\end{gathered}
$$

and the equations of motion (1) for the whole column can be written in matrix form as follows:

$$
\begin{gathered}
\mathbf{T}=\mathbf{M}_{a} \ddot{\mathbf{u}}-\mathbf{A}_{g} \\
\mathbf{M}=\mathbf{G T}+\mathbf{I}_{G} \ddot{\mathbf{u}}-\mathbf{B}_{g}
\end{gathered}
$$

where vectors $\mathbf{T}$ and $\mathbf{M}$ are nodal shear forces and moments from node $i$ to $n$ : $\mathbf{T}=\left[T_{1} T_{2} \ldots\right.$ $\left.T_{n}\right]^{T}, \mathbf{M}=\left[\begin{array}{llll}M_{1} & M_{2} & \ldots & M_{n}\end{array}\right]^{T}$; vector $\ddot{\mathbf{u}}$ is horizontal accelerations from node 2 to $n+1$ : $\ddot{\mathbf{u}}=\left[\ddot{u}_{2} \ddot{u}_{3} \ldots \ddot{u}_{n+1}\right]^{T} . \mathbf{M}_{a}, \mathbf{G}$, and $\mathbf{I}_{G}$ can be defined, respectively, as mass coefficient matrix, geometric coefficient matrix, and polar inertia coefficient matrix. $\mathbf{A}_{g}$ is a vector characterized by null values except the first component, representing the acceleration at the base of the column: $\mathbf{A}_{g}=\left[\begin{array}{lllll}a_{g}(t) m_{1} / 2 & 0 & \ldots & 0 & 0\end{array}\right]^{T}$; and, similarly, $\mathbf{B}_{g}=\left[\begin{array}{lllll}a_{g}(t) I_{G 1} / h_{1} & 0 & \ldots & 0 & 0\end{array}\right]^{T}$. Substituting the 
expression of $\mathbf{T}$ in the expression of $\mathbf{M}$, a system of differential equations to be solved for determining the displacements of the multi-drum column is obtained:

$$
\mathbf{M}=\mathbf{G M}_{a} \ddot{\mathbf{u}}-\mathbf{G A}_{g}+\mathbf{I}_{G} \ddot{\mathbf{i}}-\mathbf{B}_{g}
$$

where $\mathbf{M}=\mathbf{M}(\boldsymbol{\theta})$, since each moment $M_{i}$ is assumed to depend on the rotation $\theta_{i}$ of the corresponding $i$-th drum by means of a bi- or tri-linear moment-rotation constitutive law, which represents the stabilizing moment for varying block rotation (Figure 2), and it is slightly modified with respect to Housner law by means of an initial elastic bending stiffness $K_{M, i}$ and a smoothing parameter $\xi \leq 1$ for reducing the stabilizing moment $M_{u, i}$ at each interface:

$$
M_{u, i}=\left(B_{i} / 2\right) \sum_{j=i}^{n} P_{j}
$$

The system of differential equations in (5) is solved by means of a Runge-Kutta ODE solver.
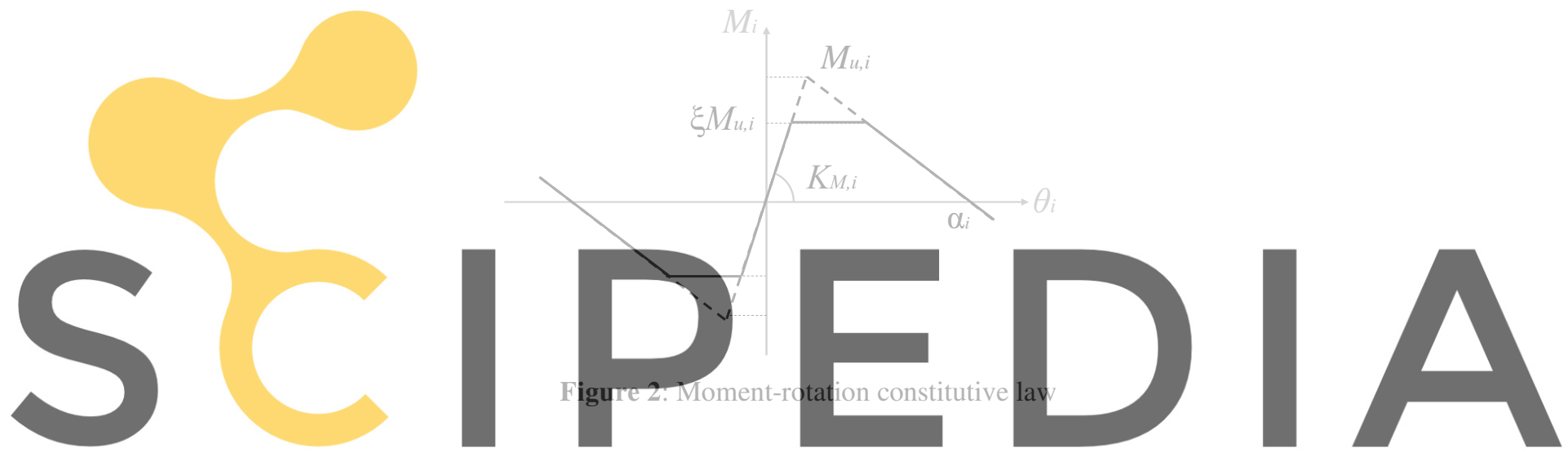

3 NUMERICAL TESTS

Register for freerat httes / wwww scipedianfem ifo dewnload the version without the watermark

Rigid Beam Model in simulating the dynamic behavior of freestanding monolithic and multidrum columns, with respect to existing numerical and analytical solutions. For instance, the numerical tests performed by Spanos and Koh [7] on simple rectangular monolithic blocks subjected to harmonic excitations were considered. Then, the slender column type already adopted by authors in past contributions [4-5] was taken into consideration and a simple halfsine wave excitation and a harmonic one were applied to the ground in order to evaluate the columns' dynamic behavior modelled with Rigid Beams and compared both with Housner solution and numerical results obtained from DEM.

\subsection{Monolithic blocks and columns subjected to harmonic excitations}

The numerical tests performed by Spanos and Koh [7] on monolithic rectangular columns were considered in this study. A slender and a thick column, having height to width ratio $H / B$ equal to 4 and 2, were subjected to harmonic excitations with varying input frequency and acceleration amplitude. Such specimens were reproduced by means of the proposed Rigid Beam Model and considering a single rigid beam element. Harmonic excitations were applied for 10 seconds. Starting with the slender column case, Figure 3 shows several deformed configurations 
at the end of the numerical tests and Figure 4 collects base and top horizontal displacements versus time. Both the deformed configurations and the horizontal displacements at the top show overturning conditions obtained with $0.5 \mathrm{~g}$ and $0.5 \mathrm{~Hz}$ and $1 \mathrm{~Hz}$. More information about the possible overturning of the column are collected in Figure 5, which shows the safe-unsafe domain obtained with the Rigid Beam Model compared with that obtained by Spanos and Koh [7], who assumed in this case a restitution coefficient close to 1 . Probably due to this condition, the two safe-unsafe-domains are in excellent agreement, both starting with a collapse acceleration equal to $0.25 \mathrm{~g}$, corresponding to the static load multiplier for overturning the column, and with an increasing collapse acceleration for increasing frequency, up to $1.5 \mathrm{~g}$ with $2 \mathrm{~Hz}$.
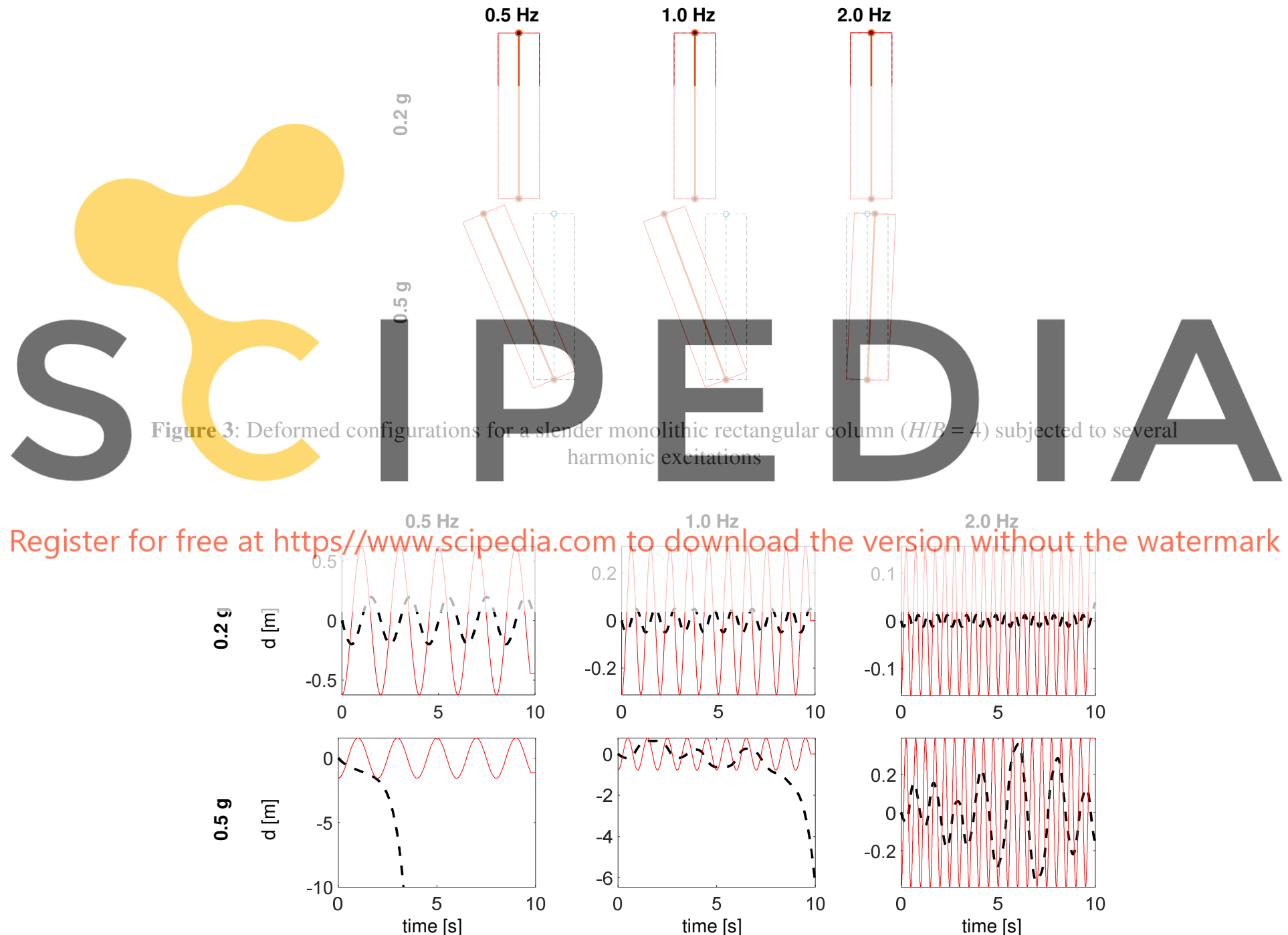

Figure 2: Base (red continuous line) and top (black dashed line) displacements for a slender monolithic rectangular column $(H / B=4)$ subjected to several harmonic excitations 


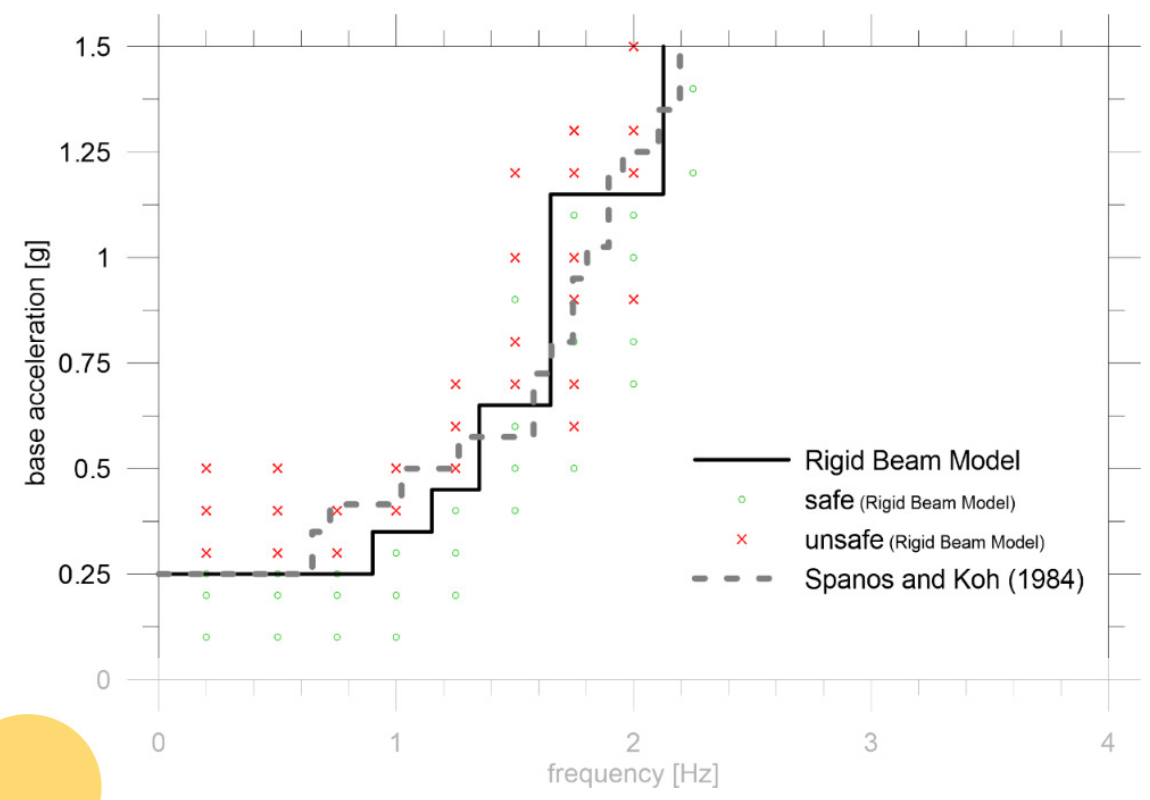

Figure 3: Safe-unsafe domain for a slender monolithic column $(H / B=4)$ subjected to harmonic excitations

Focusing then on the thick column case modelled with the proposed Rigid Beam Model, Figure

6 shows the deformed configurations at the end of the harmonic tests with several values of acceleration and frequency. Also Figure 7 shows the column top and base displacements for the same frequency and acceleration values as before. Overturning obtained with $0.5 \mathrm{~g}$ and $1 \mathrm{~Hz}$ and with $1.0 \mathrm{~g}$ with
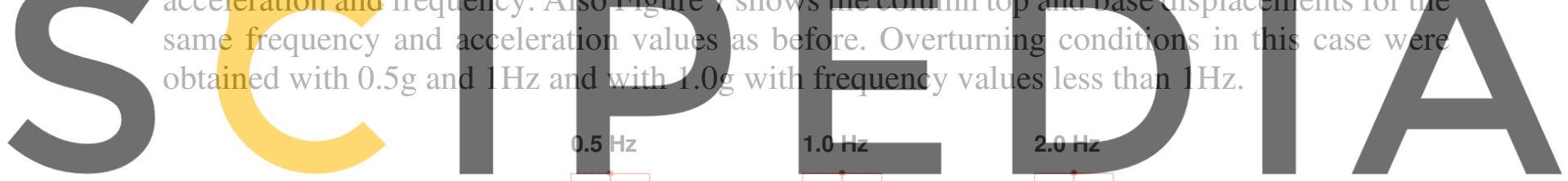

Register for free at https// Ạww.scipedia.com to download the version without the watermark
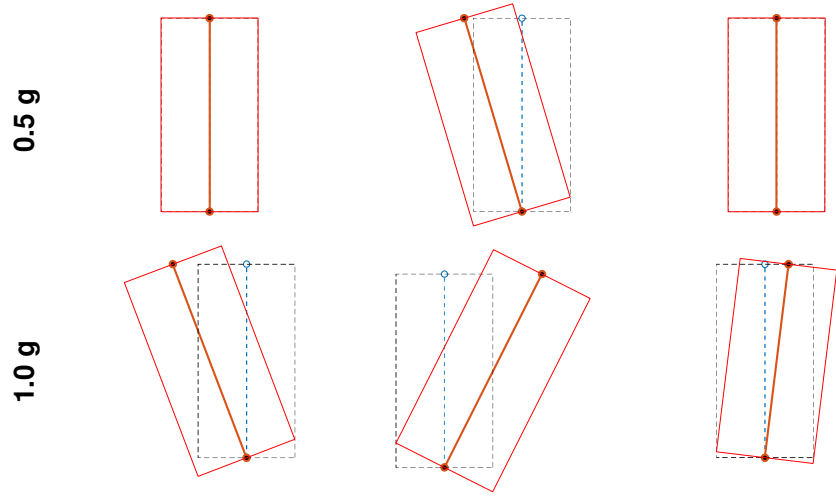

Figure 6: Deformed configurations for a thick monolithic rectangular column $(H / B=2)$ subjected to several harmonic excitations 

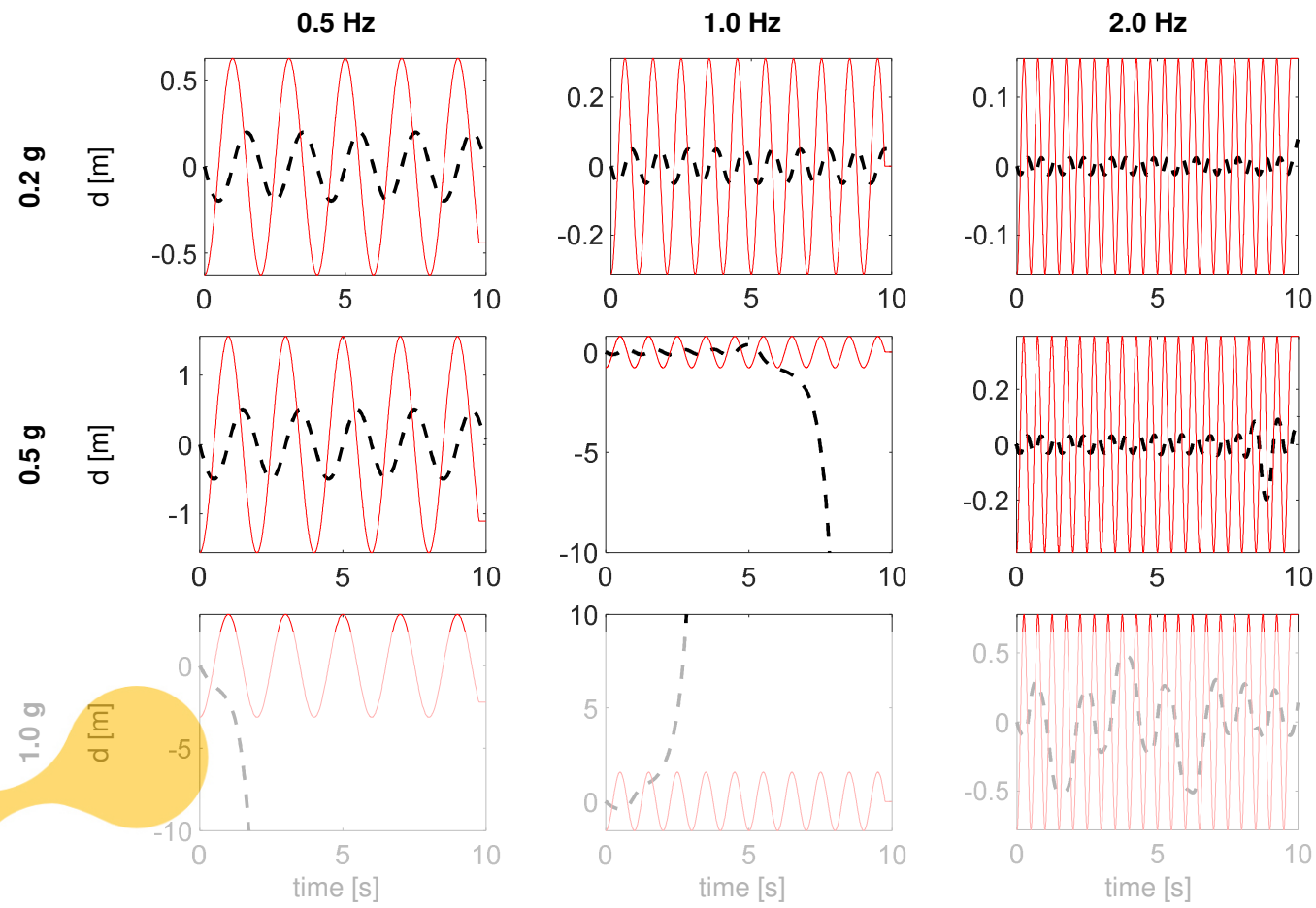

Figure 7: Base (red continuous line) and top (black dashed line) displacements for a thick monolithic rectangular
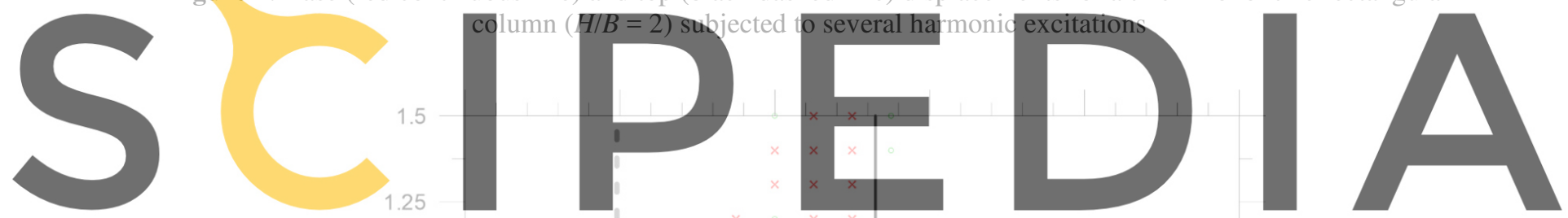

Register for free at htaps//www.scipedia.com to download the version without the watermark

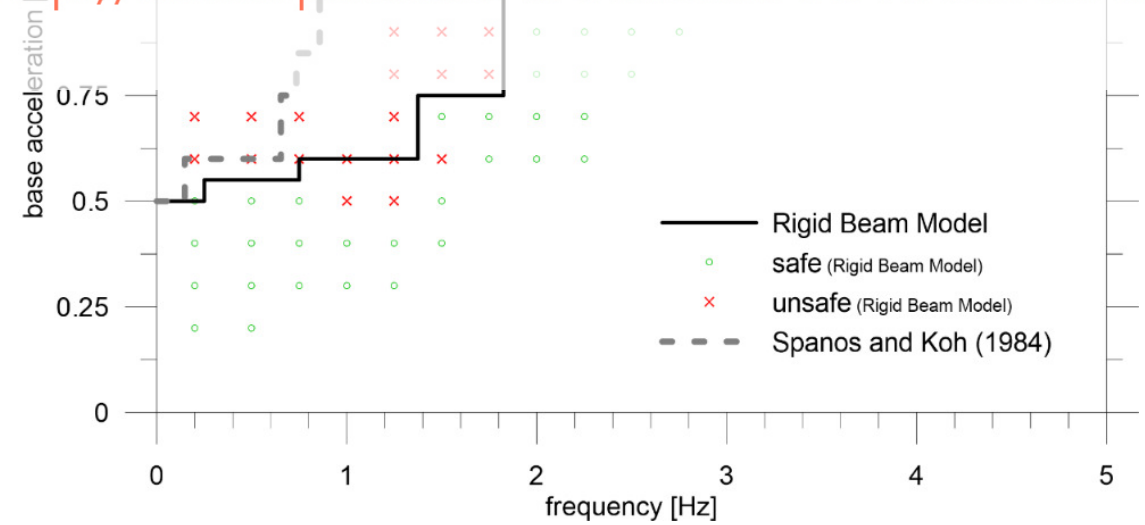

Figure 8: Safe-unsafe domain for a thick monolithic column subjected to harmonic excitations

Figure 8 shows the safe-unsafe domain obtained with the Rigid Beam Model, which is significantly different with respect to that obtained by Spanos and Koh [7], probably due to the small $H / B$ ratio of the case study considered, and also due to the restitution factor close to 0.5 
adopted in the reference work. In this case, both domains are characterized by a collapse acceleration equal to $0.5 \mathrm{~g}$ for small frequency values, which is correctly coincident with the static collapse multiplier of the thick column. For increasing frequency, the Rigid Beam Model allows to obtain overturning accelerations increasing to $1 \mathrm{~g}$ with $2 \mathrm{~Hz}$ and $1.5 \mathrm{~g}$ close to $3 \mathrm{~Hz}$, whereas the collapse acceleration values obtained by Spanos and Koh increase rapidly to $1.5 \mathrm{~g}$ close to $1 \mathrm{~Hz}$.

\subsection{Monolithic columns subjected to half-sine wave excitations}

The slender monolithic column already considered by authors in the previous research [4-5] is here adopted for further validating the proposed Rigid Beam Model. The column has an overall height $H=5 \mathrm{~m}$, and lower and upper base width $B_{1}=0.96 \mathrm{~m}, B_{n}=0.66 \mathrm{~m}$. A simple half sine wave base excitation is applied to column base by varying the amplitude and the wavelength of the excitation. Overturning accelerations obtained with the proposed model are compared with the well-known Housner solution [1]. For this purpose, the smoothing parameter of the moment-rotation relationship is assumed equal to 1, together with an infinite bending interface stiffness between the ground and the column.

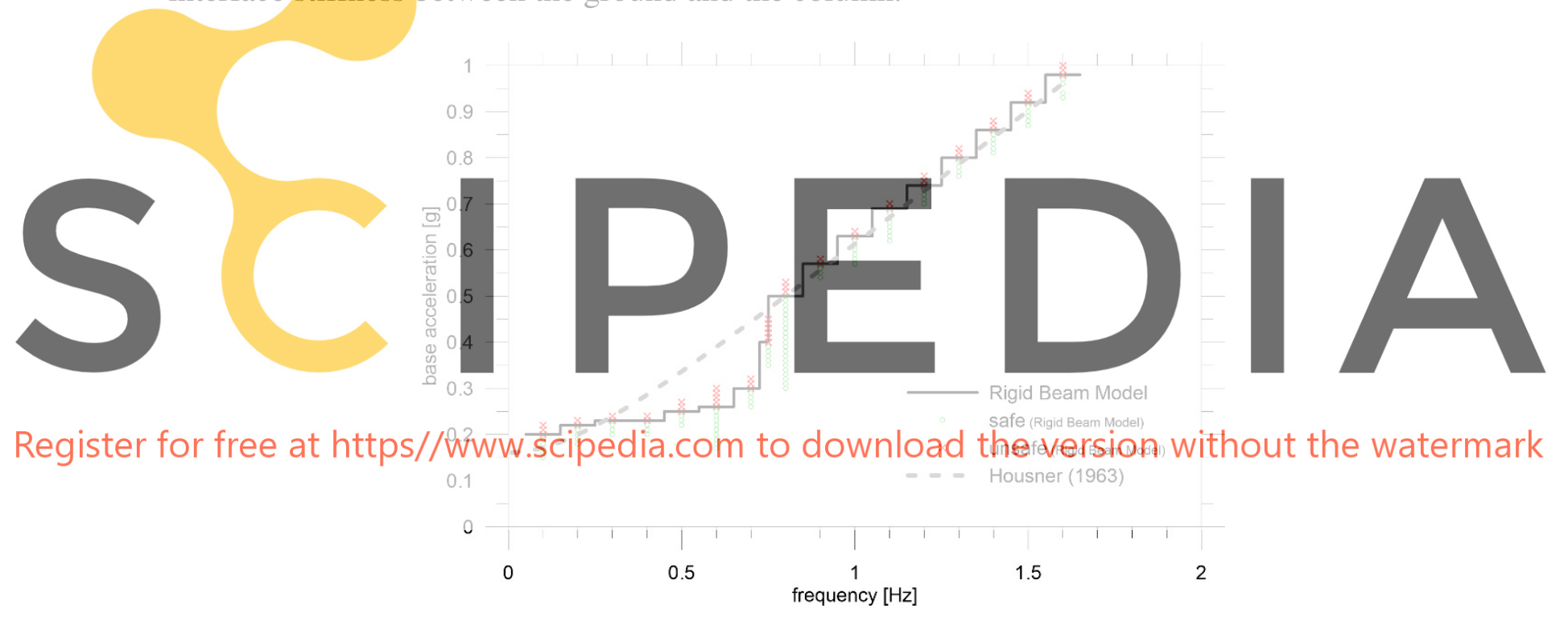

Figure 9: Safe-unsafe domain for a slender monolithic column subjected to half sine-wave excitations

Numerical results obtained with the Rigid Beam Model are in excellent agreement with Housner solution, except for a frequency range between 0.3 and $0.7 \mathrm{~Hz}$, characterized by smaller collapse accelerations obtained numerically with respect to the analytic solution. These results are collected in Figure 9.

\subsection{Monolithic columns subjected to harmonic excitations}

The numerical tests on the slender monolithic column already considered by authors are here updated by adding the column configurations at the end of the analyses or close to the end obtained with the Rigid Beam Model. Collapse mechanisms are evident since they are characterized by large rigid rotations of the column with respect to its base (Figure 10). 


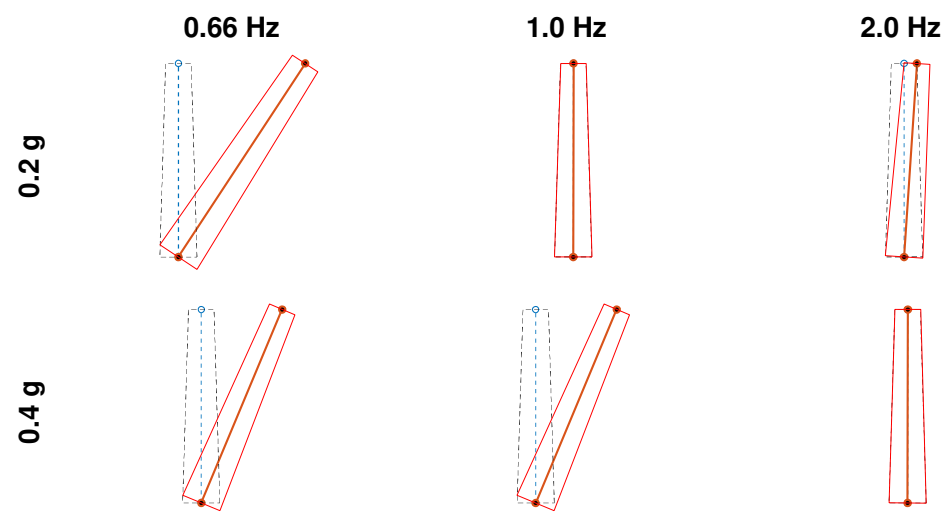

Figure 10: Deformed configurations for a monolithic column subjected to several harmonic excitations

The safe-unsafe domain obtained with the Rigid Beam Model, compared with that obtained with a standard DEM which allows both sliding and rocking of interfaces, shows that the hypothesis of no interface sliding influences the dynamic behavior of a monolithic column, since it reduces the collapse acceleration values for increasing input frequency (Figure 12a).

\subsection{Multi-drum columns subjected to harmonic excitations}

Similarly to the previous sub-section, the numerical tests on the slender multi-drum column equivalent to the monolithic one already considered by authors the column configuratio Beam Model. Collapse rotations of an upper part of the colmon or of the entire colum 11). The safe-tunsafe domain obtained with the
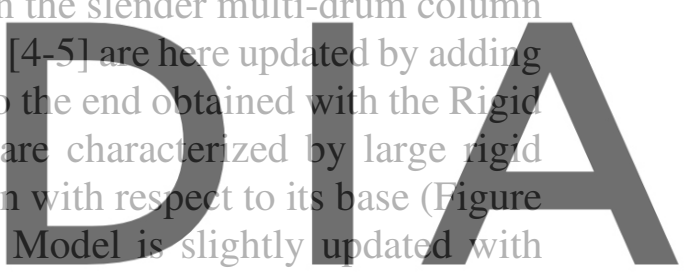
further results with respect to the previous proposed results; however, the multi-drum column

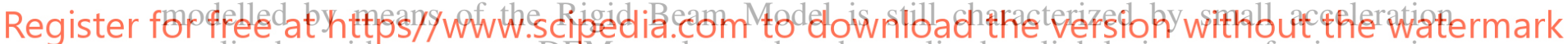
amplitudes with respect to DEM results, and such amplitudes slightly increase for increasing input frequency (Figure 12b).

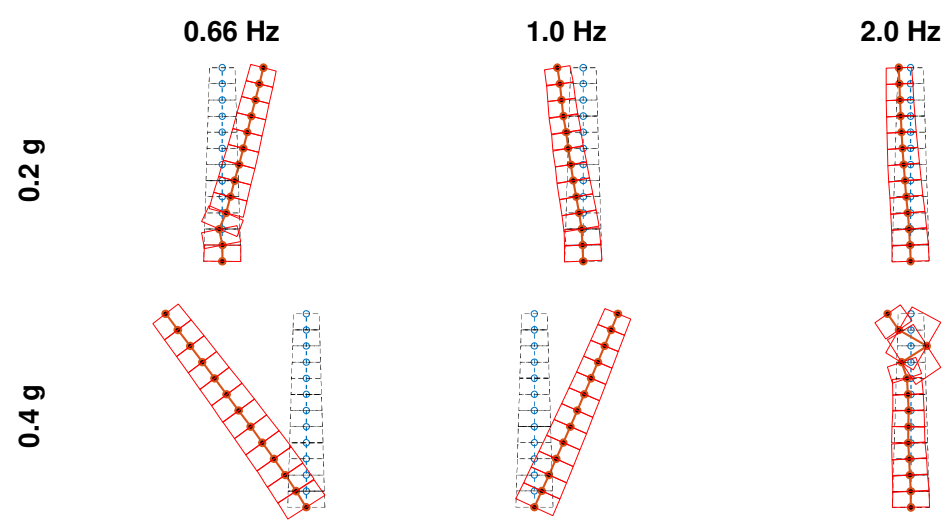

Figure 11: Deformed configurations for a multi-drum column subjected to several harmonic excitations 

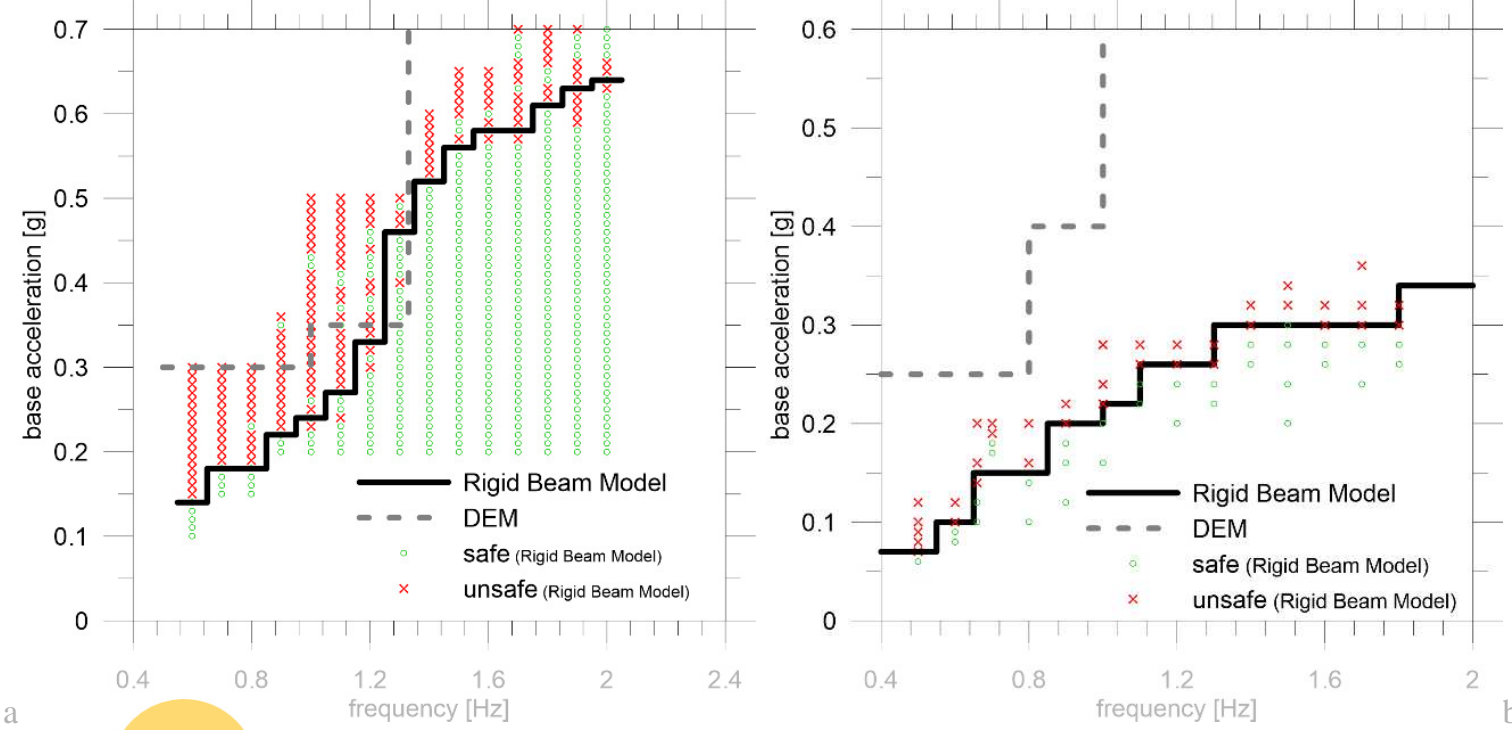

Figure 12: Safe-unsafe domain for a slender monolithic column (a) and an equivalent multi-drum column (b) subjected to harmonic excitations

4 CONCLUSIONS

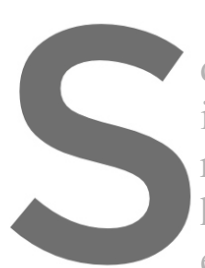

A simple and effective Rigid Beam Model

dynamic behayior of fr

investigated with severa

rigid beam element and each joint betyeen the

horizontal degrees of fre

edom of the

each joint is also assumed, whereas sliding is
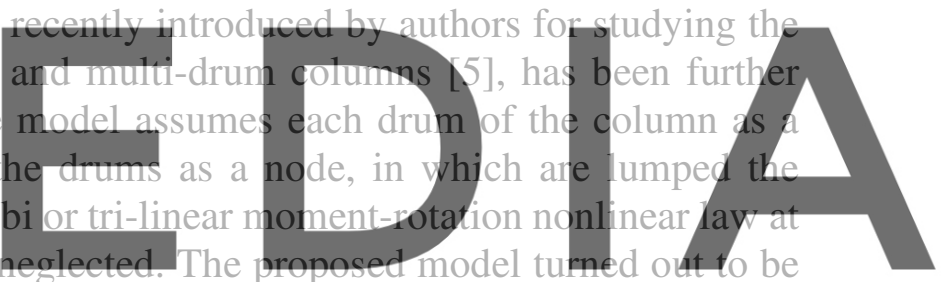

in excellent agreement with the results of the harmonic analyses performed by Spanos and Koh

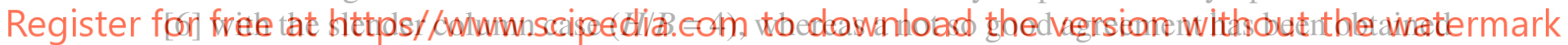

with the thick column case $(H / B=2)$, probably due to the small restitution factor adopted in the

reference work. The simple case of a ground excitation given by a half sine-wave proposed by

Housner [1] has been successfully simulated by the proposed model. Finally, the sufficient agreement of the Rigid Beam Model with respect to the DEM, already showed by authors [5], has been further detailed by performing more harmonic tests on the multi-drum column.

Further developments of the proposed Rigid Beam Model will regard the further comparisons with existing numerical and laboratory results, by considering in some cases real ground motions on monolithic and multi-drum columns.

\section{REFERENCES}

[1] Housner, G.W. The behavior of inverted pendulum structures during earthquakes. Bull. Seismol. Soc. Am. (1963) 53(1):403-417.

[2] Psycharis, I.N., Papastamatiou, D.Y. and Alexandris, A.P. Parametric investigation of the stability of classical columns under harmonic and earthquake excitations. Earthq. Eng. 
Struct. Dyn. (2000) 1(29):1093-1109.

[3] Papantonopoulos, C., Psycharis, I.N., Papastamatiou, D.Y., Lemos, J.V. and Mouzakis, H.P. Numerical prediction of the earthquake response of classical columns using the distinct element method. Earthq. Eng. Struct. Dyn. (2002) 1(31):1699-1717.

[4] Sarhosis, V. Baraldi, D., Lemos, J. and Milani, G. Dynamic behaviour of ancient freestanding multi-drum and monolithic columns subjected to horizontal and vertical excitations. Soil Dyn. Earthq. Eng. (2019) 120:39-57.

[5] Baraldi, D., Milani, G. and Sarhosis, V. Numerical models for simulating the dynamic behaviour of freestanding ancient columns. Compdyn 2019 Proceedings (2019), pp. 15261536.

[6] Cundall, P.A., A computer model for simulating progressive large scale movements in blocky rock systems. Proceedings of the Symposium of the International Society of Rock Mechanics (1971), Nancy, France.

[7] Spanos, P.D. and Koh, A.-S. Rocking of rigid blocks due to harmonic shaking. J. Eng. Mech. (1984) 110(11):1627-1642.

[8] Itasca. UDEC - Universal Distinct Element Code manual: theory and background. Minneapolis, Itasca Consulting Group, (2004). 